\title{
Connaissances et perceptions des producteurs sur la gestion des parcs à Vitellaria paradoxa C. F. Gaertn. (karité) au Burkina Faso
}

\author{
Hermann Yempabou OUOBA ${ }^{1 *}$, Brigitte BASTIDE ${ }^{2}$, Pascaline COULIBALY-LINGANI ${ }^{3}$, \\ Sibiry Albert KABORE ${ }^{1}$ et Joseph Issaka BOUSSIM ${ }^{1}$ \\ ${ }^{1}$ LaBEV/UO1 - 09 BP 848 Ouagadougou 09, Burkina Faso. \\ ${ }^{2}$ DEF/INERA - Station de Farako Bâ - BP 910 Bobo-Dioulasso, Burkina Faso. \\ ${ }^{3}$ DEF/INERA - 03 BP 7047 Ouagadougou 03, Burkina Faso. \\ *Auteur correspondant ; E-mail : herman249@yahoo.fr; Tel. : 0022670676075
}

\section{REMERCIEMENTS}

Les auteurs remercient le Fond International pour la Science (FIS) et le Programme de Productivité Agricole en Afrique de l'Ouest (PPAAO/WAAPP) pour le financement de cette étude.

\section{RESUME}

Au Burkina Faso, les producteurs appliquent un système intégré d'utilisation des terres qui associe l'arbre aux cultures et à l'élevage dénommé parc agroforestier. Notre objectif est d'évaluer la perception et les savoirs des producteurs sur la conservation et le renouvellement des arbres des parcs agroforestiers en général et des parcs à karité en particulier. L'échantillonnage probabiliste stratifié a été utilisé sur les différentes populations dans sept sites, réparties le long d'un gradient climatique nord-sud. Ainsi, trois groupes ont été retenus par site à savoir 10 femmes non propriétaires terriennes, 10 hommes non propriétaires terriens et 10 hommes propriétaires terriens. Les résultats montrent que les producteurs conservent préférentiellement 32 espèces ligneuses dont Vitellaria paradoxa C. F. Gaertn. qui est largement la plus citée (98\%). Les cultures vivrières et de rente associées aux arbres sont au nombre de 18. Quelle que soit la localité, les principales catégories d'utilisation des arbres conservés sont l'alimentation, l'économie, la pharmacopée et l'ombrage. Cependant, les difficultés rencontrées dans la gestion des parcs agroforestiers sont au nombre de 19. Malgré toutes les contraintes biotiques et abiotiques de gestion efficiente, le parc agroforestier demeure un système important de gestion de la phytodiversité présentant de multiples intérêts socio-économiques et écologiques. (C) 2018 International Formulae Group. All rights reserved.

Mots clés : Vitellaria paradoxa C. F. Gaertn., parc agroforestier, ethnobotanique, savoirs locaux, Burkina Faso. 


\title{
Farmers Knowledge and perceptions of management of Vitellaria paradoxa $\mathrm{C}$. F. Gaertn. (shea tree) parkland in Burkina Faso
}

\begin{abstract}
In Burkina Faso, farmers are implementing integrated land use systems that combine the tree with crops and livestock called parkland. The objective of our work was to evaluate the perception and the knowledge of the farmers about the conservation and the renewal of the trees of the parkland in general and particularly of the shea trees parkland. Seven localities divided along a climatic gradient were investigated. Stratified probability sampling based on gender and property status was used on the different populations for ethno-botanical surveys. At each site, three groups were discriminated: 10 women non-landowners, 10 men non-landowners and 10 men landowners. Results show that farmers preferentially conserve 32 woody species in their parklands. Among these species, Vitellaria paradoxa C. F. Gaertn. occupies the first position (approximately 98\%). Crops associated with trees are 18. Among 11 categories of use, the main are food, economy, pharmacopoeia and shade in all localities. The difficulties encountered by the producers in parkland' management are 19. Despite constraints for efficient management, parklands remain an important system for managing plant biodiversity with multiple socio-economic and ecological interests.
\end{abstract}

(c) 2018 International Formulae Group. All rights reserved.

Keywords: Vitellaria paradoxa C. F. Gaertn., parkland, ethnobotanic, farmers knowledge, Burkina Faso.

\section{INTRODUCTION}

Depuis les dernières décennies, plusieurs espèces végétales de grand intérêt socio-culturel, économique et écologique pour les populations rurales sont en déclin du point de vue de leur densité au Burkina Faso. Ce phénomène est lié à la péjoration des conditions climatiques et à la surexploitation des ressources végétales (Traoré et al., 2011 ; Yaméogo et al., 2011). Ainsi, une menace plane sur la sécurité alimentaire, économique et culturelle des communautés locales fortement dépendantes et peut conduire à terme à la disparition de ressources phytogénétiques (Belem, 2009). Dans ces régions, depuis des siècles, les producteurs appliquent un système intégré d'utilisation des terres qui associe l'arbre aux cultures et à l'élevage. Ce système se caractérise par le maintien d'arbres sélectionnés et épargnés lors des défrichements pour la mise en culture qui sont donc disséminés dans l'espace agraire (Smektala et al., 2005).
Dénommé «parc agroforestier », il constitue le résultat d'un long processus de transformation du milieu par des techniques d'aménagement capitalisées sur plusieurs générations et généralement maîtrisées du point de vue écologique, agronomique et socio-économique où afin de compenser la diminution de la jachère, les producteurs utilisent souvent la rotation des cultures.

Au Burkina Faso, malgré les nombreuses études se rapportant aux espèces préférées par les populations rurales (Mertz et al., 2001; Kristensen et Balslev, 2003 ; Lykke et al., 2004 ; Belem et al., 2008), les besoins et les priorités des populations locales en matière d'arbres sont peu connus, ce qui limite la mise en œuvre de programmes efficients de développement agricole et forestier participatif et durable (Belem et al., 2008). Cependant, il s'avère que Vitellaria paradoxa C. F. Gaertn., très apprécié pour la matière grasse tirée de ses amandes, est citée parmi les ligneux les plus préservés dans les parcs agroforestiers des 
zones semi-arides de l'Afrique sub saharienne du Sénégal à l'Ouganda (Lovett et Haq, 2000). Kaboré et al. (2012) notent qu'il est l'espèce préférée des paysans de la zone soudanienne du Burkina Faso du fait de l'importance des ressources financières générées par la vente de ses amandes. En fonction des régions, la densité des peuplements de Vitellaria paradoxa C. F. Gaertn. est supérieure ou égale à 30 pieds à l'hectare (War, 2007) soit environ 195 millions de pieds dans le pays (Thiombiano et al., 2016). Le karité a fourni 28,991 milliards de FCFA soit $0,60 \%$ de la valeur du PIB courant du Burkina Faso en 2011 (IAP, 2011 in MEED, 2012) alors que la contribution globale de l'ensemble des PFNL à l'économie nationale du Burkina avait été évaluée à $0,63 \%$ pour l'année 2008 (IPE, 2011 in MEED, 2012).

Partant de ces faits et de la nécessité d'impliquer les agriculteurs dans la recherche de solutions pour la pérennisation du système des parcs agroforestiers, nous avons jugé nécessaire d'effectuer un diagnostic de leurs perceptions et connaissances de la gestion de ces parcs. Ainsi, l'objectif visé par cette étude est d'évaluer la perception et les savoirs des producteurs sur la conservation et le renouvellement des arbres des parcs agroforestiers en général et des parcs à karité en particulier. Les objectifs spécifiques de cette étude sont les suivantes :

- identifier les facteurs socioéconomiques et socio-culturels qui déterminent le choix des espèces végétales conservées dans les parcs agroforestiers;

- déterminer la perception des producteurs sur la densité du parc agroforestier ;

- appréhender les connaissances endogènes sur la gestion durable des parcs agroforestiers.

\section{MATERIEL ET METHODES Zone d'étude}

Sur l'ensemble du territoire burkinabé, le système de production agricole est marqué par une faible mécanisation, des difficultés d'accès aux intrants et un manque de formation des producteurs concourant à de faibles rendements. De ce fait, les populations rurales utilisent des organes végétaux ligneux ou non conservées dans les parcs agroforestiers comme complément alimentaire et source de revenus pour compenser les faibles récoltes.

Le Burkina Faso comprend le long d'un gradient climatique une végétation variée formant quatre secteurs phytogéographiques et un large spectre d'intensité d'utilisation des terres selon Fontès et Guinko (1995) (Figure 1). Les mêmes auteurs notent que la physionomie de la végétation dans le secteur sub sahélien est steppique comportant des espèces du genre Acacia Mill. et des espèces soudaniennes très ubiquistes comme Combretum glutinosum Perr. ex DC. Le secteur nord soudanien est très peuplé et la savane y présente régulièrement un paysage agreste de type parc dominé par des arbres appartenant aux espèces protégées agro-forestières telle que Vitellaria paradoxa C. F. Gaertn. Le secteur sud soudanien comporte les formations forestières les plus denses du pays. Il présente un fond floristique soudanien commun auquel sont associées des espèces ripicoles guinéennes telle que Cola laurifolia Mast.

Le climat burkinabé est caractérisé par une saison sèche et une saison pluvieuse contrastées, la saison sèche s'étalant sur 5 à 10 mois suivant un gradient climatique nordsud. Selon les données de la météorologie nationale du Burkina Faso, les précipitations moyennes annuelles de 1991 à 2015 vont de $532 \pm 82 \mathrm{~mm}$ dans le secteur sub sahélien à $1150 \pm 149 \mathrm{~mm}$ dans le secteur sud soudanien en passant par $860 \pm 109$ dans le secteur nord soudanien (Figure 2).

Les sites d'étude couvrent sept localités qui sont: Sillia et Bouria (secteur sub sahélien); Gonsé et Kodjonti (secteur nord soudanien) ; Sobaka, Noumoudara et Kakoumana (secteur sud soudanien). Leur localisation ainsi que les caractéristiques 
démographiques et socio-économiques de leurs populations sont renseignées dans le Tableau 1. Le choix des sites d'étude est fonction de l'aire de répartition du karité au Burkina Faso et du plan de mise en œuvre du projet dénommé: valorisation en milieu paysan des acquis de la recherche pour lutter contre la dégradation et le vieillissement des peuplements de karités en fonction de la zone climatique au Burkina Faso (Bouria, Gonsé et Noumoudara). Selon Orwa et al. (2009) l'espèce est présente dans les savanes humides et sèches où les précipitations annuelles oscillent entre 500 et $1400 \mathrm{~mm}$ à l'exception des zones marécageuses ou soumises à une longue période d'inondation.

\section{Echantillonnage et collecte des données}

Dans chaque site, un

échantillonnage probabiliste stratifié basé sur le genre et le statut de propriété a été utilisé sur les différentes populations pour les enquêtes ethnobotaniques. L'approche genre se justifie par les usages plus ou moins différents que font les femmes et les hommes des arbres (Kristensen et Lykke, 2003). Quant au statut de propriétaire, il détermine la gestion durable, en l'occurrence la dynamique à long terme des ligneux du parc. Sur chaque site, trois groupes ont été discriminés à savoir 10 femmes non propriétaires terriennes, 10 hommes non propriétaires terriens et 10 hommes propriétaires terriens. Seuls les adultes ont été interviewés du fait que Lamy Lamy et al. (2018) considèrent que l'âge et le sexe présentent un intérêt capital car ils impactent sur les stratégies locales de gestion des ressources naturelles. L'entretien semi structuré a été conduit individuellement avec des producteurs qui sont les premiers responsables de la gestion de leurs parcelles. Le questionnaire a porté essentiellement sur les caractéristiques socio-économiques des informateurs, les pratiques culturales, le sens donné à la densité des arbres dans les parcs, les difficultés et la description des procédés de gestion durable des parcs. Les espèces citées ont été vérifiées grâce à la technique du guide voyageur (Albuquerque et al., 2006) qui comporte 3 étapes : la notation des noms locaux des espèces connues et citées par l'informateur ; l'accompagnement d'un individu de la population dans l'habitat naturel de la plante citée pour une observation directe ; la détermination du nom scientifique de l'espèce par utilisation d'une flore. Les noms scientifiques suivent la nomenclature adoptée par Thiombiano et al. (2012). Ces auteurs mentionnent aussi la concordance des noms moré et dioula qui nous a permis de reconnaitre les noms scientifiques correspondants.

\section{Traitement et analyse des données}

Les caractéristiques démographiques et socio-économiques de la population permettant de mieux appréhender les pratiques et connaissances des informateurs ont été d'abord recensées. Les fréquences relatives (FR) de citation des espèces ligneuses conservées préférentiellement dans les parcs agroforestiers et celles des cultures vivrières et de rentes ont été calculées. Une analyse de variance (ANOVA) des différentes fréquences relatives de citation des espèces ligneuses a été effectuée. Le calcul des FR a servi aussi pour apprécier les usages regroupés en catégories ainsi que les points de vue des producteurs sur la densité moyenne des arbres. Nous avons obtenu onze catégories d'utilisation que sont l'alimentation, l'économie, la pharmacopée, l'ombrage, la fertilisation des sols, le fourrage, la protection contre l'érosion, le bois de feu, le bois d'œuvre, la culture et l'augmentation de la pluviométrie.

$\mathrm{FR}=\frac{\mathrm{n}}{\mathrm{N}} \times 100$

n: le nombre de fois que l'espèce a été citée ;

$\mathbf{N}$ : le nombre total d'informateurs ;

Les contraintes objectives et réellement vécues par les producteurs ont été mises en évidence à partir de difficultés mentionnées par les producteurs dans chacun des sites et les fréquences relatives de citation des différentes difficultés ont été calculées. Le seuil de $20 \%$ a été retenu pour distinguer 
les catégories d'utilisation et les difficultés les plus citées de celles les moins citées (Tiétiambou et al., 2016).

Une analyse en composante principale (ACP) a permis de regrouper les différentes localités en fonction des menaces qui pèsent sur les parcs à karité. L'objectif de l'ACP est de mettre en évidence graphiquement l'information essentielle d'un tableau de données ; son grand mérite tient à ce qu'elle permet de voir plus clairement et simultanément l'ensemble des variables.

Une hiérarchisation des savoirs paysans dans la gestion des parcs à karité a été établie à partir du nombre de pratiques de gestion efficiente des parcs agroforestiers connu par les producteurs. Pour ce faire nous avons considéré la moyenne obtenue par chaque groupe d'interviewés repartis par localité, sexe et statut de propriété. Les pratiques citées par les interviewés étant au nombre de 22. Le test paramétrique du « Khideux » a été utilisé pour les comparaisons entre localité.

Les données brutes ont été synthétisées dans le logiciel Microsoft Excel 2016 pour le calcul des fréquences relatives de citation. Une fréquence d'information n'est considérée significative, que lorsqu'elle est supérieure ou égale à $20 \%$ de l'ensemble de l'échantillon enquêté pour une question donnée (PRPW/ECOPAS, 2002). Le même logiciel a permis de générer des graphiques d'illustration. Le logiciel JMP 12.1.0 a servi pour l'ANOVA à un facteur et le logiciel IBM SPSS Statistics v20 a permis d'effectuer l'ACP. Les différents logiciels cités sont des programmes permettant un traitement des données statistiques.
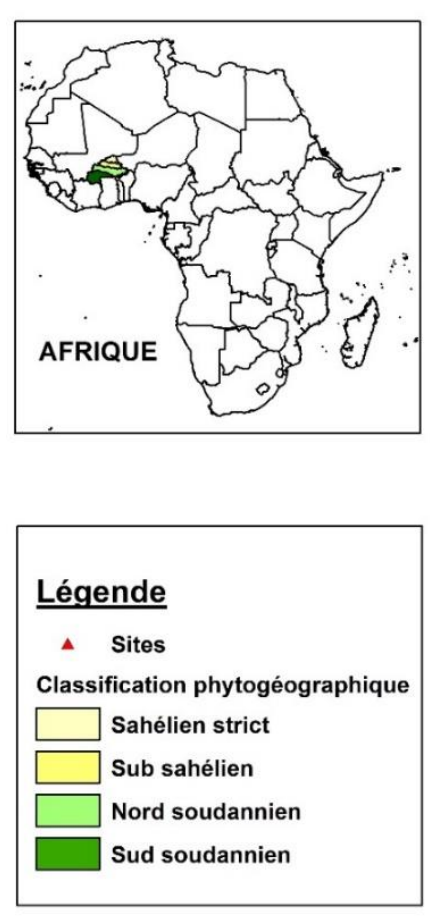

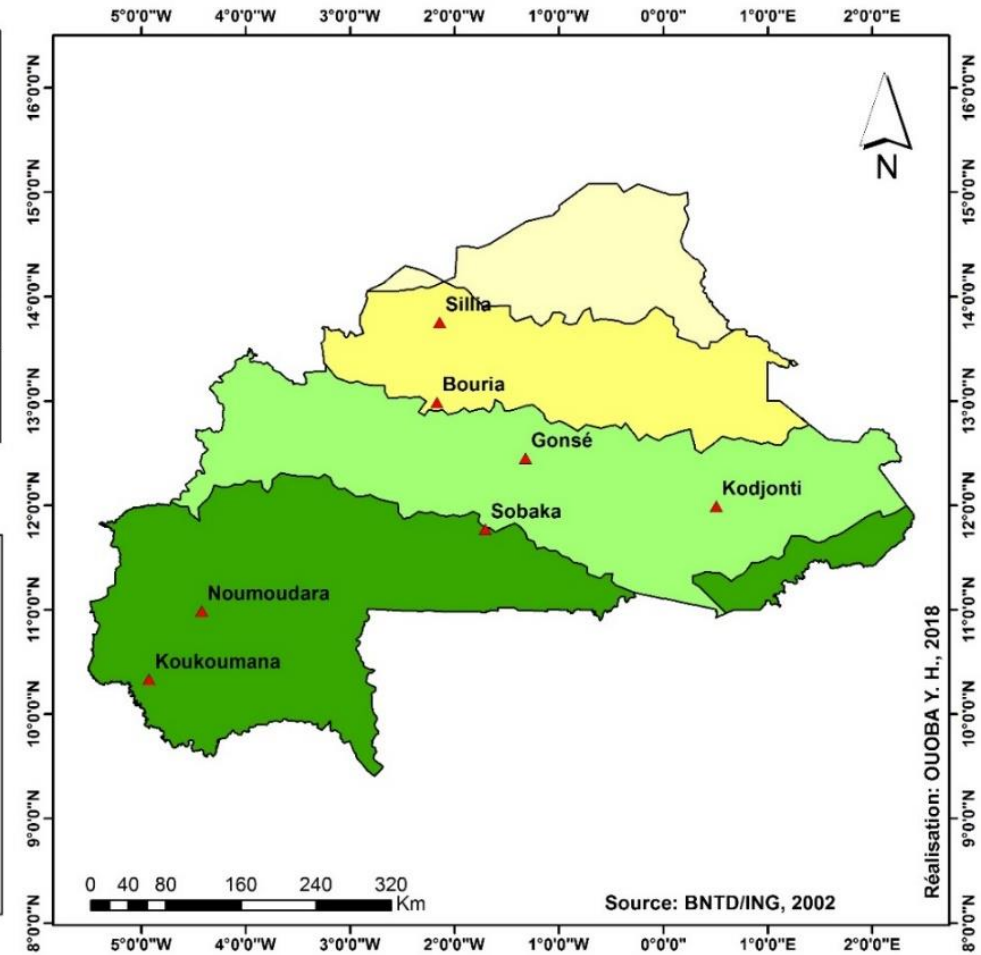

Figure 1 : Localisation des sites d'étude. 


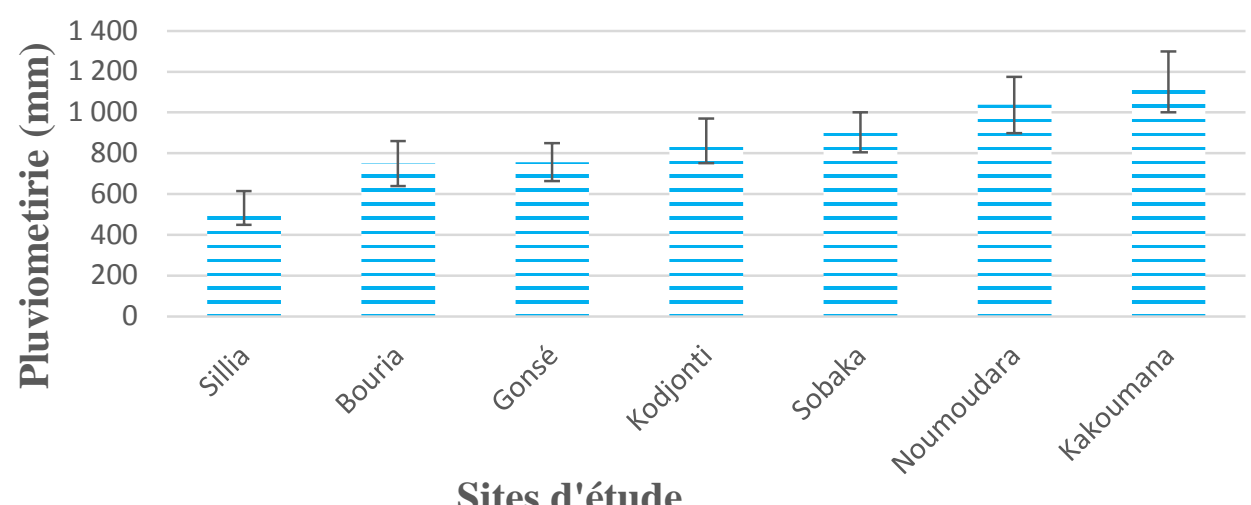

Figure 2 : Valeurs moyennes de précipitations dans les sites d'étude entre 1991 et 2015.

Tableau 1: Caractéristiques démographiques et socio-économiques des populations des sites d'étude.

\begin{tabular}{lllll}
\hline $\begin{array}{l}\text { Sites d'étude } \\
\text { (Villages) }\end{array}$ & Communes & Populations & $\begin{array}{l}\text { Ethnies } \\
\text { majoritaires }\end{array}$ & $\begin{array}{l}\text { Principales } \\
\text { cultures }\end{array}$ \\
\hline Sillia & Titao & 466 & Mossi & Niébé/Sorgho blanc \\
\hline Bouria & Yako & 204 & Mossi & Niébé/Sorgho blanc \\
\hline Gonsé & Saaba & 101 & Mossi & Niébé/Sorgho blanc \\
\hline Kodjonti & Fada N'Gourma & 222 & Gourmantché & Sorgho rouge/Niébé \\
\hline Sobaka & Sapouy & 821 & Mossi & Niébé/Sorgho blanc \\
\hline Noumoudara & Péni & 655 & Tiéfo & Maïs/Niébé \\
\hline Kakoumana & Niangoloko & 283 & Gouin & Maïs/Niébé \\
\hline
\end{tabular}

\section{RESULTATS}

\section{Caractéristiques générales des} informateurs

Conformément aux critères de stratifications, la proportion des hommes est plus élevée que celle des femmes soit $66,7 \%$. Il en est de même pour le statut de propriété qui présente une proportion de $66,7 \%$ de non propriétaires terriens. Les personnes d'âge compris entre 30 et 60 ans constituent la classe d'âge majoritaire avec une fréquence de 60,9\%. Par ailleurs, le nombre de personnes mariées est très élevé $(88,1 \%)$ par rapport aux célibataires qui sont minoritaires $(1,4 \%)$. Le reste de la population est représenté par les veufs/veuves $(10,5 \%)$. Outre le fait que la majorité des informateurs $(67,1 \%)$ soient des migrants, 53,8\% appartiennent au groupe ethnique Mossi. Il faut aussi noter qu'une forte proportion $(68,6 \%)$ des informateurs est analphabète. La plupart des informateurs tirent leurs revenus de la vente d'une partie de leurs productions agricoles, des Produits Forestiers Non Ligneux (PFNL) ainsi que du bétail $(53,8 \%)$. Les tailles des exploitations sont principalement comprises entre 3 et 6 ha, avec une fréquence de $35,7 \%$.

\section{Espèces conservées préférentiellement dans les parcs agroforestiers}

L'analyse des variances entre les fréquences relatives de citation des différentes espèces montre que les producteurs conservent préférentiellement 32 espèces 
ligneuses dans leurs parcs agroforestiers sur l'ensemble des sites d'étude. Parmi ces espèces Vitellaria paradoxa C. F. Gaertn. occupe largement la première position (environ 98\%) suivie par sept autres espèces dont les fréquences relatives de citation sont supérieures à la moyenne globale des fréquences. Il s'agit de: Parkia biglobosa (Jacq.) R. Br. ex G. Don f. (néré), Lannea microcarpa Engl. \& K. Krause (raisinier), Tamarindus indica L. (tamarinier), Bombax costatum Pellegr. \& Vuill. (kapokier à fleur rouge), Balanites aegyptiaca (L.) Del. (dattier du désert), Sclerocarya birrea (A. Rich.) Hochst. (marula) et Adansonia digitata L. (baobab) (Figure 3).

Les cultures vivrières et de rente associées aux arbres dans les parcs agroforestiers sont au nombre de 18 (Figure 4). Les plus citées sont Vigna unguiculata (L.) Walp. subsp. unguiculata (niébé) (75\%), Sorghum bicolor (L.) Moench (sorgho blanc) (73\%), Zea mays L. (maïs) (72\%) et Arachis hypogea L. (arachide) (70\%).

\section{Importance des arbres dans les parcs agroforestiers en fonction du site}

Quelle que soit la localité, les principales catégories d'utilisation des arbres conservés dans les parcs agroforestiers sont l'alimentation, l'économie, la pharmacopée et l'ombrage (Figure 5). L'usage des arbres pour la fertilisation des champs est plus important à Sillia $(53 \%)$ que dans les autres sites, tandis que l'utilisation fourragère est dominante à Gonsé (33\%). Les différences sont significatives entre les différentes localités selon chaque catégorie d'utilisation $\left(\mathrm{X}^{2}=4,721 ; \mathrm{p}=1\right)$.

\section{Sens donné à la densité des parcs agroforestiers par les producteurs}

Les points de vue des producteurs sur la densité moyenne des arbres en considérant à la fois les avantages tirés des arbres et la surface laissée pour la production agricole varient énormément (Figure 6) Les opinions diffèrent en fonction de la localité, du genre et du statut de propriétaire des répondants. Une forte majorité $(46,5 \%)$ considère que cette densité est très insatisfaisante. Respectivement $16,2 \%, 17,6 \%$ et $19,5 \%$ de la population de l'étude répondent que la densité moyenne des arbres dans leurs exploitations est insatisfaisante, satisfaisante ou très satisfaisante.

Les options qui se dégagent des réponses sur le sens donné à la densité du parc agroforestier sont au nombre de trois. Dans l'ensemble, les enquêtés veulent augmenter la densité actuelle des arbres dans leurs exploitations $(83,3 \%)$. Le maintien de cette densité est souhaité par 13,3\% et seulement $3,3 \%$ de la population veulent la diminuer (Figure 7). Cette dernière part de la population est composée à $75 \%$ par les enquêtés de Sobaka et de Kakoumana (zone sud soudanienne). Par ailleurs, les points de vue diffèrent en fonction de la localité, du genre et du statut de propriété des répondants.

\section{Difficultés rencontrées dans la gestion des parcs}

Les difficultés rencontrées par les producteurs dans la gestion des parcs agroforestiers sont au nombre de 19. Les principales difficultés sont par ordre d'importance : les feux de brousse $(47,1 \%)$, la divagation des animaux $(46,2 \%)$ et la sècheresse $(35,7 \%)$ (Figure 8$)$. Nombre de menaces sont d'origine anthropique comme les pratiques culturales néfastes et la coupe abusive; d'autres face auxquelles l'agriculteur reste relativement impuissant ont été citées, tel que le vieillissement des arbres et les vents.

Le nombre de menaces qui pèsent sur la pérennité des parcs à karité varie énormément d'une localité à une autre (Figure 9). Le plan engendré par les deux composantes de l'ACP montre une nette opposition d'une part entre les zones à climat plus arides (à gauche du graphique) avec les différentes menaces qui y sévissent dont la sécheresse et la divagation des animaux; et la zone sud soudanienne (à droite du graphique) avec les feux et les pratiques culturales néfastes. 
Les parcs agroforestiers de Sillia, Bouria, Gonsé et Kodjonti sont les plus soumis à l'impact de la sécheresse, de la coupe abusive, de la divagation des animaux, de l'érosion, de l'écorçage, des ravageurs et des termites. A Sobaka, les menaces majeures sont les Loranthacées, les vents violents, la pression foncière, la collecte intégrale des amandes, la faible fertilité des sols et l'indisponibilité des plants de karité chez les pépiniéristes pour effectuer les plantations. Dans les localités de Noumoudara et de Kakoumana, les producteurs sont préoccupés par le manque de mise en jachère, les feux, les éléphants qui déracinent les arbres, les pratiques culturales néfastes telles que l'utilisation d'herbicides et de tracteur, le manque de main d'œuvre pour protéger la régénération naturelle ou planter et le vieillissement des peuplements.

\section{Connaissances endogènes de gestion durable des parcs agroforestiers}

Les producteurs ont des savoirs endogènes de gestion des parcs à karité, au total 22 types ont été recensés. Il s'agit principalement de la Régénération Naturelle Assistée -RNA- (96\%), la surveillance des arbres $(63 \%)$ et la plantation $(60 \%)$.

Le nombre total et le nombre moyen des savoirs endogènes ont été regroupés en fonction de la localité, du sexe et du statut de propriété (Tableau 2). En termes d'effectifs de savoirs, les non propriétaires terriens dépassent les propriétaires terriens de 4 , tandis que les hommes dépassent les femmes de 3 savoirs. Pour ce qui est du nombre moyen de savoirs par personne, les propriétaires terriens $(4,9 \pm 1,5)$ dépassent les non propriétaires $(4,4$ $\pm 1,4)$ tandis que les hommes $(4,8 \pm 1,5)$ surpassent les femmes $(4 \pm 1,1)$. La moyenne des savoirs de la population étant de 4,6 $\pm 1,4$. D'une localité à l'autre les nombres de savoirs varient de 12 (Sillia) à 18 (Gonsé et Sobaka) mais les moyennes varient entre $5,4 \pm 1,3$ (Gonsé) et 3,9 $\pm 1,5$ (Kakoumana). Les différences sont significatives entre les différentes localités pour ce qui est du nombre de savoir $\left(X^{2}=3,807 ; p=0,9999991\right)$. Les différences entre les nombres moyens de savoirs également sont significatives entre les différentes localités $\left(\mathrm{X}^{2}=0,391 ; \mathrm{p}=1\right)$.

Tableau 2 : Nombre total et nombre moyen des savoirs endogènes par producteur regroupés par localité, par sexe et en fonction du statut de propriété.

\begin{tabular}{|c|c|c|c|c|c|c|c|c|c|c|c|}
\hline & \multicolumn{5}{|c|}{$\begin{array}{c}\text { Nombre total de } \\
\text { savoirs }\end{array}$} & \multicolumn{5}{|c|}{ Nombre moyen de savoirs par producteur } \\
\hline & & \begin{tabular}{|l|}
$\mathbf{E}$ \\
\end{tabular} & H & $\mathbf{F}$ & $\mathbf{P}$ & $\mathbf{N P}$ & $\mathbf{E}$ & $\mathbf{H}$ & $\mathbf{F}$ & $\mathbf{P}$ & $\overline{\mathbf{N P}}$ \\
\hline \multirow{7}{*}{ Localités } & Sillia & 12 & 12 & 5 & 6 & 12 & $4,8 \pm 0,9$ & $4,8 \pm 1$ & $4,7 \pm 0,5$ & $4,8 \pm 1$ & $4,8 \pm 0,8$ \\
\hline & Bouria & 17 & 16 & 12 & 12 & 16 & $4,4 \pm 1,5$ & $4,7 \pm 1,7$ & $3,8 \pm 1$ & $5,1 \pm 1,9$ & $4 \pm 1,2$ \\
\hline & Gonsé & 18 & 16 & 11 & 13 & 14 & $5,4 \pm 1,3$ & $5,6 \pm 1,3$ & $5 \pm 1$ & $5,8 \pm 1,1$ & $5,2 \pm 1,3$ \\
\hline & Kodjonti & 15 & 15 & 8 & 9 & 14 & $4,5 \pm 1,5$ & $4,8 \pm 1,6$ & $3,8 \pm 1$ & $4,7 \pm 1,2$ & $4,4 \pm 1,6$ \\
\hline & Sobaka & 18 & 15 & 12 & 12 & 16 & $4,8 \pm 1,4$ & $5,3 \pm 1,2$ & $3,7 \pm 1,2$ & $5,8 \pm 0,8$ & $4,3 \pm 1,4$ \\
\hline & Noumoudara & 16 & 14 & 11 & 13 & 14 & $4,2 \pm 1,4$ & $4,5 \pm 1,4$ & $3,6 \pm 1,4$ & $4,2 \pm 0,9$ & $4,2 \pm 1,7$ \\
\hline & Kakoumana & 14 & 12 & 8 & 11 & 10 & $3,9 \pm 1,5$ & $4 \pm 1,8$ & $3,6 \pm 0,5$ & $4,4 \pm 2,4$ & $3,6 \pm 0,7$ \\
\hline & $\mathrm{E}$ & 22 & 18 & 15 & 17 & 21 & $4,6 \pm 1,4$ & $4,8 \pm 1,5$ & $4 \pm 1,1$ & $4,9 \pm 1,5$ & $4,4 \pm 1,4$ \\
\hline
\end{tabular}

Légende : $\mathrm{E}=$ ensemble, $\mathrm{H}=\mathrm{Homme}, \mathrm{F}=\mathrm{Femme}, \mathrm{P}=$ Propriétaire, $\mathrm{NP}=$ Non Propriétaire. 


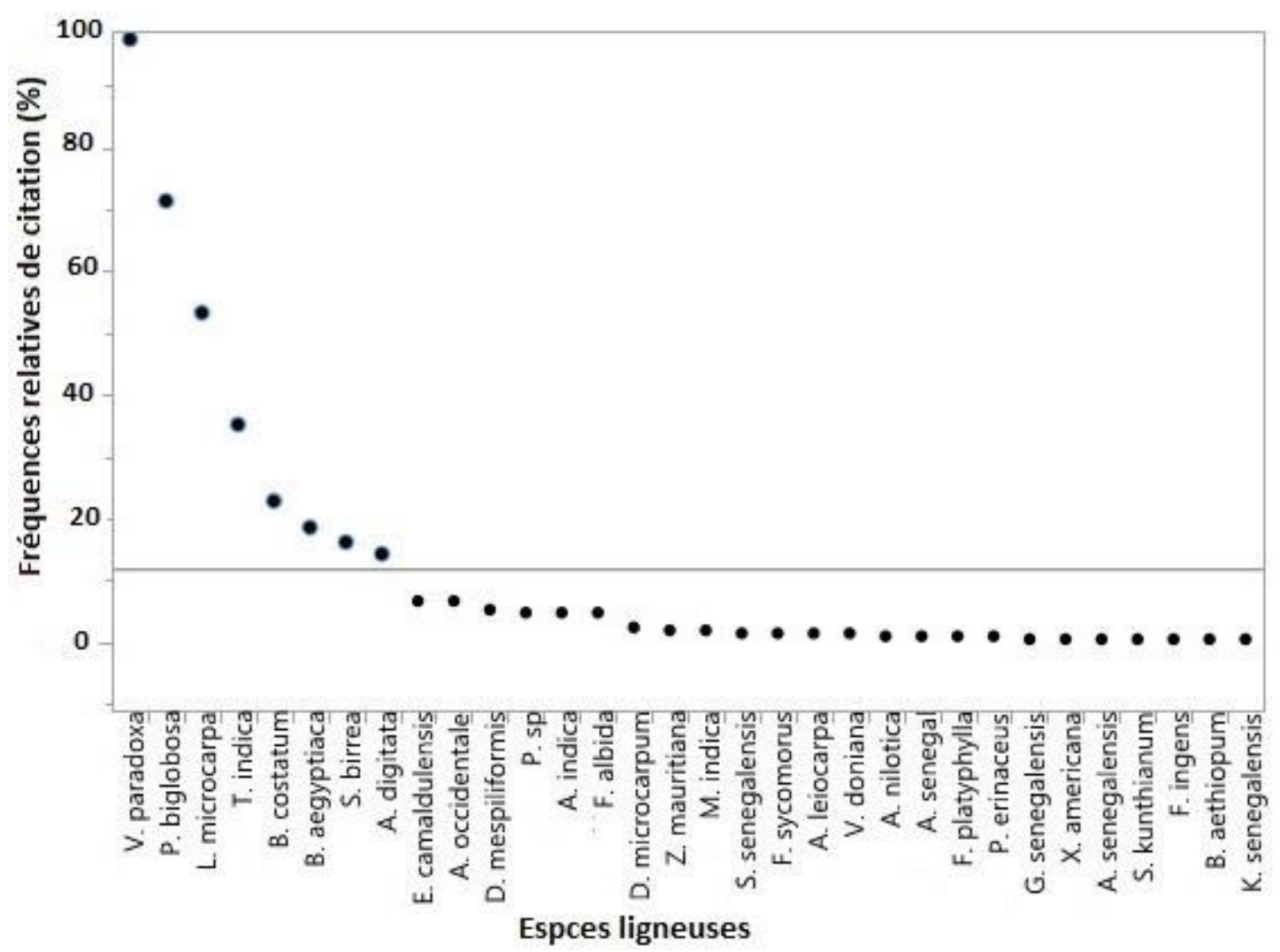

Figure 3 : Classification des espèces ligneuses conservées préférentiellement dans les parcs agroforestiers selon leurs fréquences relatives de citation.



Figure 4 : Classification des cultures associées aux arbres dans les parcs agroforestiers selon leurs fréquences relatives de citation. 


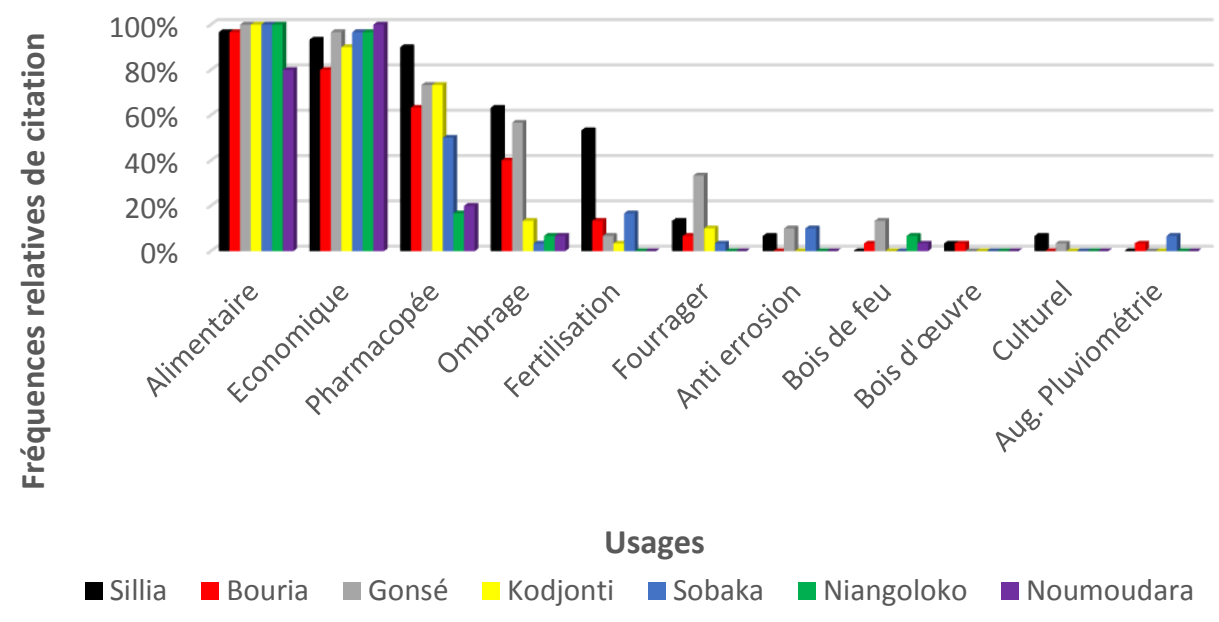

Figure 5 : Importance de chaque catégorie d'utilisation par localité.

Ensemble Homme Femme Propriétaire $\begin{gathered}\text { Non } \\ \text { propriétaire }\end{gathered}$

Figure 6 : Fréquence des opinions sur les avantages et inconvénients de la densité actuelle des arbres dans les exploitations.
Légende :
=très insatisfaisante
= insatisfaisante
$=$ satisfaisante
$=$ très satisfaisante 


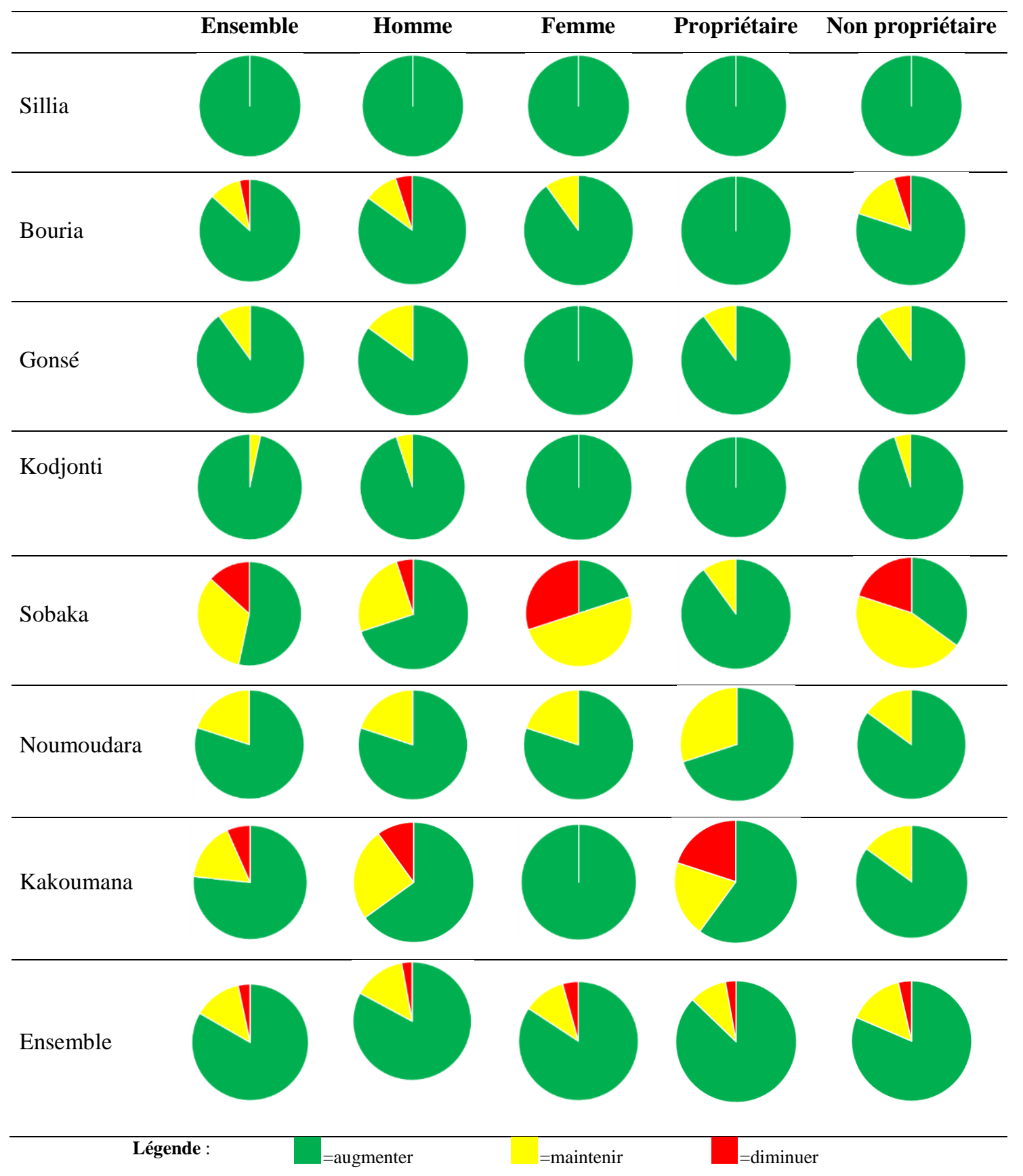

Figure 7 : Fréquence des opinions sur le sens donné à la densité du parc agroforestier. 


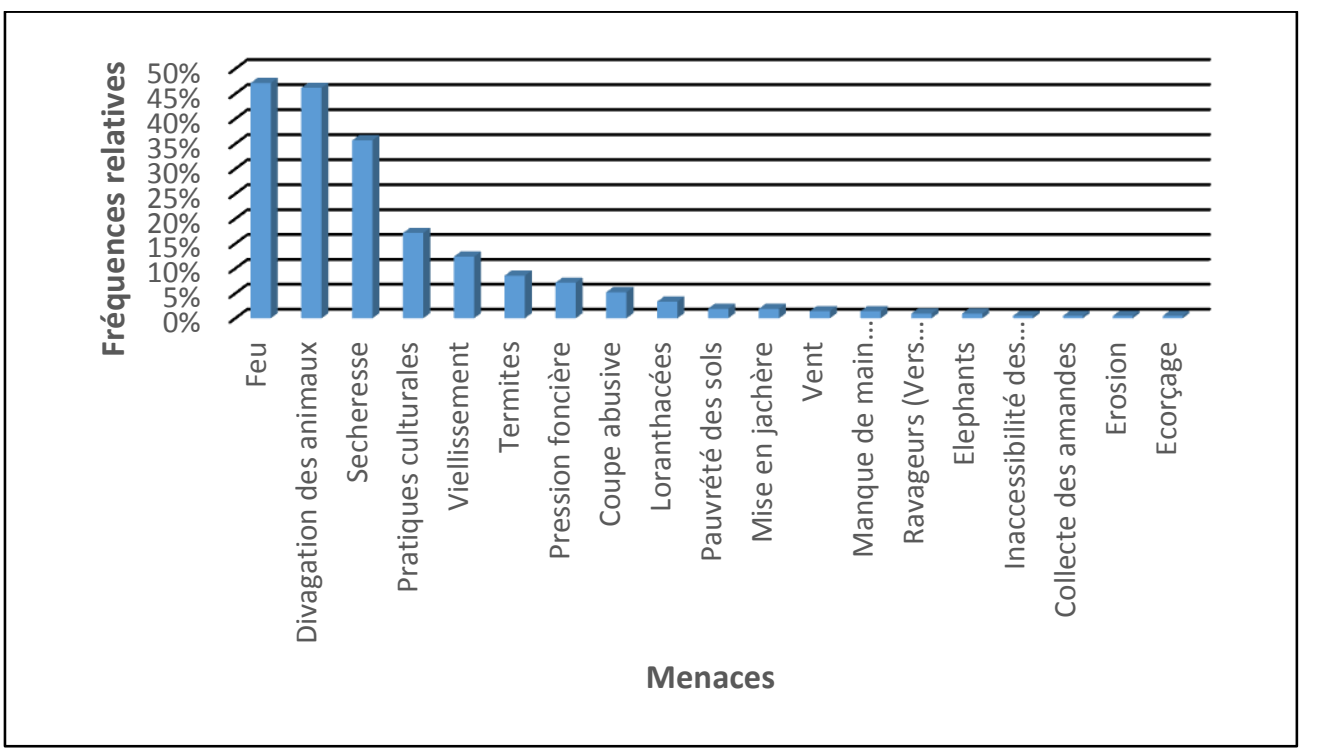

Figure 8 : Fréquences relatives de citation des menaces qui pèsent sur les parcs agroforestiers.

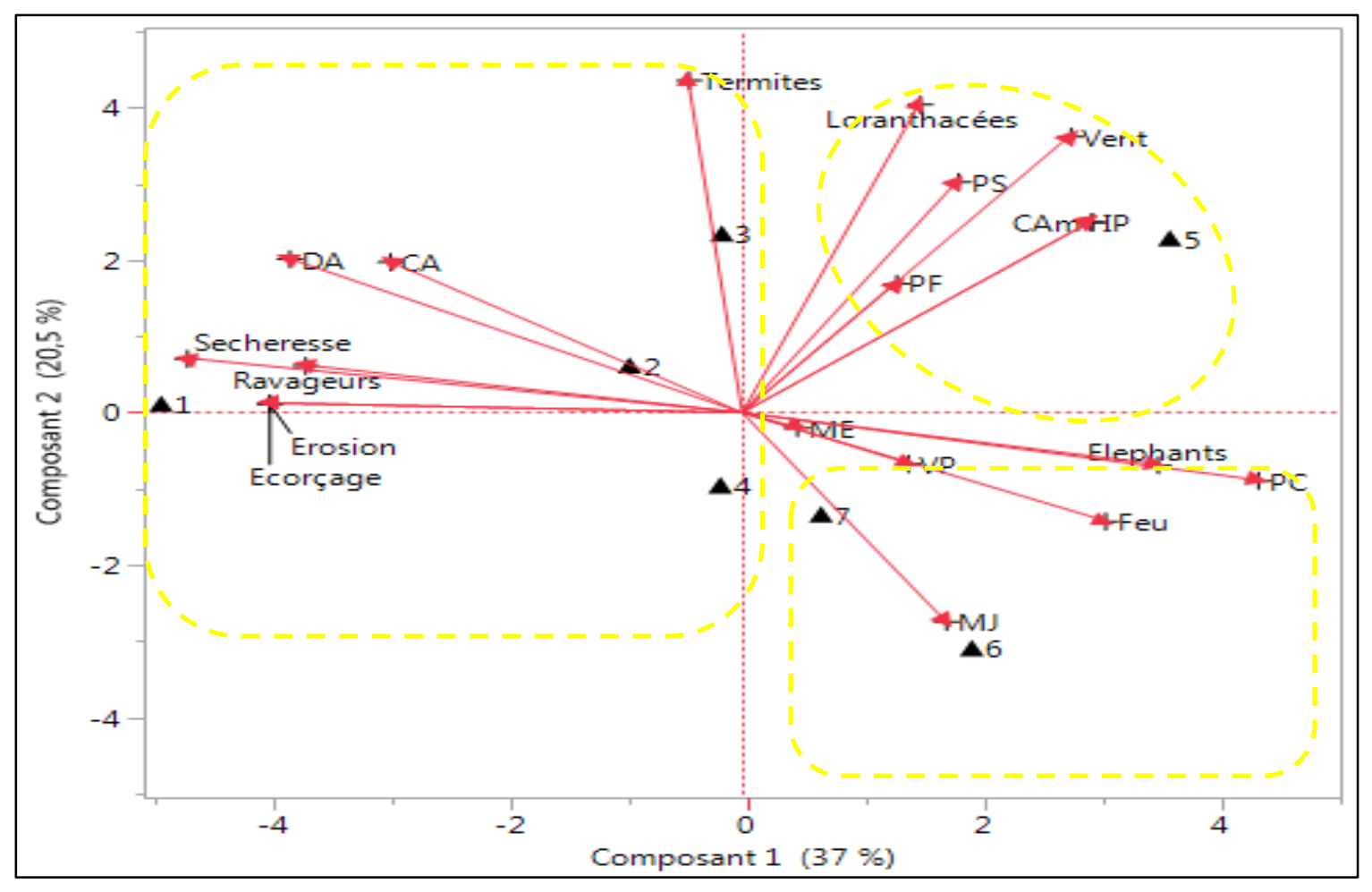

Figure 9 : Corrélations entre les menaces qui pèsent sur les parcs à karité et les localités.

Légende : Sillia $=1$, Bouria $=2$, Gonsé $=3$, Kodjonti $=4$, Sobaka $=5$, Kakoumana $=6$, Noumoudara $=7, \mathrm{CA}=$ coupe abusive, $\mathrm{DA}=$ divagation des animaux, $\mathrm{ME}=$ manque de main d'œuvre, $\mathrm{MJ}=$ manque de mise en jachère, $\mathrm{PC}=$ pratiques culturales néfastes, $\mathrm{PF}=$ pression foncière, $\mathrm{CAm}=$ collecte intégrale des amandes, $\mathrm{PS}=$ pauvreté des sols, $\mathrm{IP}=$ inaccessibilité des plants de karité, $\mathrm{VP}=$ vieillissement des peuplements. 


\section{DISCUSSION}

\section{Espèces conservées préférentiellement dans les parcs agroforestiers}

La liste des espèces végétales pérennes conservées préférentiellement dans les parcs agroforestiers au Burkina Faso est relativement similaire à celles rapportées par divers auteurs (Kristensen et Lykke, 2003 ; Lykke et al., 2004 ; Belem et al., 2008). Le karité est cité comme le principal arbre utilitaire conformément à Yaméogo et al. (2005) qui ont noté qu'en terme de nombre de pieds conservés, l'espèce occupe la première place, suivie par Parkia biglobosa (Jacq.) R. Br. ex G. Don f. Selon Kaboré et al. (2012) le karité est également l'espèce préférée des paysans de la zone sud-soudanienne du Burkina Faso du fait de l'importance des ressources financières générées par la vente de ses amandes. Coulibaly-Lingani et al. (2011), ont aussi noté que parmi les produits forestiers non ligneux, ce sont les amandes de karité et les graines de Néré qui offrent les revenus les plus élevés. Au Bénin, Gnanglè et al. (2012) ont rapporté l'importance de ces parcs à karité et à Néré dans les paysages agrosylvicoles comme sources de revenus pour des populations locales et d'un intérêt pour la conservation de l'environnement.

D'après Boffa et al. (2000) et Kristensen et Balslev (2003), les principales cultures vivrières et de rente associées aux arbres sont le petit mil, le sorgho, le maïs et le coton. En plus de ces cultures, il faut inclure le niébé et l'arachide. Le système de production des parcs est caractérisé par une rotation des cultures dans les différentes parcelles de l'exploitation ainsi qu'une alternance entre la mise en culture et la mise en jachère dont les délais varient énormément. Le but de ces pratiques est de reconstituer la fertilité du sol.

\section{Importance des arbres dans les parcs agroforestiers en fonction du site}

Nos résultats montrent que le parc agroforestier revêt un grand intérêt pour les producteurs, en ce sens qu'il est pourvoyeur d'espèces hautement utilitaires dans divers domaines. Il s'agit principalement de l'alimentation (Lannea microcarpa Engl. \& K. Krause ; Tamarindus indica L.), l'économie (Vitellaria paradoxa C. F. Gaertn.; Parkia biglobosa (Jacq.) R. Br. ex G. Don f.), la pharmacopée (Azadirachta indica A. Juss.; Detarium microcarpum Harms) et l'ombrage (Ficus sycomorus L. subsp. gnaphalocarpa (Miq.) C.C. Berg ; Diospyros mespiliformis Hochst. ex A. DC.). Plusieurs études ont aussi prouvé que les espèces les plus conservées sont les plus utiles et par conséquent les plus préférées des producteurs (Kristensen et Balslev, 2003 ; Yaméogo et al., 2005).

Le nombre d'usage ainsi que les critères de sélection des arbres dans les parcs diffèrent très peu quelle que soit la localité, mais leur degré d'importance varie d'une localité à l'autre. Ces divergences dans le choix des espèces à conserver seraient induites par des disparités socioculturelles (Atato et al., 2010).

\section{Sens donné à la densité des parcs agroforestiers par les producteurs}

La densité des arbres dans les parcs agroforestiers est considérée comme étant très insatisfaisante par $46,7 \%$ des enquêtés quel que soit le site à cause des biens et services que les arbres leur procurent. A l'opposé, $19,5 \%$ des producteurs trouvent que cette densité est très satisfaisante. Ce fait s'explique en premier lieu par l'insuffisance des terres cultivables face à une démographie en constante augmentation. Dans l'ensemble, les producteurs établissent une corrélation entre le 
rendement et la superficie exploitée sans tenir compte du matériel et des intrants agraires ainsi que de la formation. Le constat qu'ils en font est la faiblesse des rendements qui est alors attribuée aux arbres même si leur densité est optimale. Selon Yaméogo et al. (2005), une des principales raisons de la coupe des arbres du parc est l'ombrage qui réduit par compétition la production agricole.

Le statut de propriété est le second paramètre qui conditionne l'évolution de la densité du parc agroforestier. Pour la plupart, les non propriétaires n'ont pas le droit de planter dans leurs exploitations ni de collecter les produits issus des arbres. C'est pourquoi, malgré la faible densité des arbres dans le parc, plusieurs exploitants affirment que cette densité est très satisfaisante sachant qu'ils n'ont pas le droit de planter si ce n'est que de diminuer la densité actuelle. Troisièmement, les politiques de conservation consistant à interdire la coupe, si elles ont permis le maintien des parcs, elles ont aussi eu un effet contreproductif car les agriculteurs se sont sentis dépossédés de la gestion de leur patrimoine, si bien qu'ils n'ont plus conservé ou planté de karités, d'où le vieillissement des peuplements de cette espèce karité (Smektala et al., 2005). Par ailleurs, les sites situés dans les zones les plus humides (Noumoudara et Kakoumana) présentent des parcs agroforestiers denses qui entravent parfois la production céréalière.

\section{Difficultés rencontrées dans la gestion des parcs}

Les feux de brousse tardifs ont une action négative sur la régénération car ils brûlent les jeunes plants et les organes floraux des arbres adultes réduisant ainsi leurs potentialités productives (Mapongmetsem et al., 2011). Le piétinement des bovins détruit la régénération selon Mapongmetsem et al. (2011); il en est de même pour le broutage.
Nos résultats en rapport avec l'impact de la sècheresse sur l'état des parcs à karité sont similaires à ceux de Thiombiano et al. (2016) qui ont constaté que depuis le début des années 1970, l'intensification du processus de désertification a eu des répercussions négatives au sein de ces parcs. A l'instar de Mapongmetsem et al. (2011) et de Kaboré et al. (2012); nous avons noté que le nombre réduit des plantules dans les agrosystèmes serait fortement lié aux travaux champêtres. Selon Smektala et al. (2005), le vieillissement des peuplements est dans l'ensemble consécutif au manque de régénération dû aux politiques de conservation naturelle consistant à interdire la coupe du karité. En effet, il en ressort que le manque de maintien des plantules est surtout motivé par le fait que le propriétaire de la parcelle n'a pas le droit de diminuer la densité des karités même lorsqu'elle entrave la production céréalière. Ensuite, lorsque l'exploitant de la parcelle n'est pas propriétaire, il n'a pas d'intérêt direct à conserver la régénération car dans certains cas, il n'a pas le droit de collecter les fruits des arbres qui se trouvent dans son champ dont il n'a pas la possession. Enfin, les non propriétaires terriens n'ont pas le droit de planter puisque tout acte de plantation est interprété comme une appropriation de la terre qu'ils exploitent temporairement.

\section{Connaissances endogènes de gestion durable des parcs agroforestiers}

Conformément à Yaméogo et al. (2013), nous notons que la RNA et la plantation sont les techniques d'enrichissement des parcs les plus employées par les producteurs.

De façon générale, le mode oral de transmission des savoirs rend l'information vulnérable sur le long terme tout en remettant en cause sa fiabilité (Tiétiambou et al., 2016). Cependant, selon les résultats les producteurs 
non propriétaires terriens possèdent plus de savoirs endogènes (21 savoirs) que les propriétaires terriens (17 savoirs) qui sont à 98,6\% des autochtones. Ces propriétaires terriens sont des hommes sauf dans la localité de Noumoudara où une femme, descendante des chefs de terre, possède de grandes superficies. La raison qu'on peut avancer est que les non propriétaires terriens sont des migrants qui capitalisent non seulement les savoirs de leurs localités d'origine mais aussi de ceux du site d'accueil. Par contre, la moyenne des savoirs des propriétaires est supérieure $(4,9 \pm 1,5)$ à celle des non propriétaires $(4,4 \pm 1,4)$ du fait que certains individus disposent de très peu de savoirs. En termes de genre, le nombre ainsi que la moyenne des savoirs des hommes dépassent ceux des femmes (18 pour les hommes et 15 pour les femmes). Selon Elias et Carney (2007), les femmes sont les principales détentrices des savoirs d'utilisations domestiques. Seulement, pour ce qui est de la gestion des parcs, les hommes étant les propriétaires sont également les décideurs et donc ceux qui ont le plus d'intérêts à s'informer. Les variations des connaissances entre les différentes localités sont imputables à la zone climatique étant donné que dans le sens nord-sud du gradient climatique, la moyenne des savoirs décroît. Ce fait s'explique par les difficultés de gestion du parc plus importantes dans le nord à cause de la sècheresse qui induit la faible densité des arbres. En outre, les populations du nord sont soutenues par plusieurs structures de développement qui œuvrent contre l'avancée du désert dans cette partie du pays.

\section{Conclusion}

La présente étude a permis d'identifier les espèces préférées par les populations rurales qui sont conservés dans les parcs agroforestiers au Burkina Faso ainsi que les cultures qui leur sont associées. Le karité est une espèce hautement utilitaire sur toute l'étendue de son aire de distribution au Burkina Faso. C'est l'espèce qui dessine très souvent la physionomie des parcs agroforestiers accompagnée généralement en saison pluvieuse par le niébé et le sorgho. L'alimentation, l'économie, la pharmacopée et l'ombrage représentent les principaux usages recensés qui vont de pair avec l'importance que les populations accordent aux arbres. Cette importance varie significativement selon le gradient climatique nord-sud. Dans l'ensemble, les producteurs veulent augmenter la densité des arbres dans leurs exploitations mais ils sont confrontés à des difficultés majeures qui sont entre autres le feu de brousse, la divagation des animaux et la sècheresse. Pour faire face à ces dernières, les populations rurales ont capitalisé plus d'une vingtaine de savoirs pour une gestion durable des parcs agroforestiers.

Enfin de compte, la conservation des espèces agroforestières qui nécessite leur domestication doit être une préoccupation majeure dans les politiques de gestion durable des ressources végétales visant la sécurité alimentaire et l'épanouissement socioéconomique des populations rurales. Les valeurs socio-économiques et écologiques des parcs devront être dégagées pour convaincre l'opinion publique dans la mise en œuvre de politiques d'exploitation rationnelle des PFNL et des programmes de régénération pour préserver la ressource. Pour ce faire, nous recommandons la création d'une plateforme regroupant tous les acteurs que sont les populations rurales, les industriels, les décideurs et les chercheurs.

\section{CONFLIT D'INTERETS}

Les auteurs déclarent n'avoir aucun conflit d'intérêts. 


\section{CONTRIBUTIONS DES AUTEURS}

HYO a effectué la collecte, l'analyse et le traitement des données ainsi que la rédaction de l'article; SAK s'est chargé de la revue bibliographique et de la rédaction du protocole; PC-L s'est occupée de l'affinement du questionnaire d'enquête ; BB et JIB ont assuré l'encadrement de l'étude. Tous les co-auteurs ont amandé le présent article lors de son élaboration.

\section{REMERCIEMENTS}

Les auteurs adressent leur gratitude aux populations de Sillia, Bouria, Kodjonti, Gonsé, Sobaka, Noumoudara, Kakoumana et à l'interprofession de la Table Filière Karité (TFK) pour leur disponibilité ainsi qu'aux collaborateurs scientifiques pour leur soutien.

\section{REFERENCES}

Albuquerque UP, Lucena RF, Monteiro JM, Florentino AT, Almeida CB. 2006. Evaluating two quantitative ethnobotanical techniques. Ethnobotany Research and Applications, 4: 51-60. DOI:

www.ethnobotanyjournal.org/vol4/i1547 -3465-04-051.

Atato A, Wala K, Batawila K, Woegan AY, Akpagana K. 2010. Diversité des fruitiers ligneux spontanée du Togo. Fruit, Vegetable and Cereal Sci. and Biotechnol., 4(1):1-9.

Belem B, Olsen CS, Theilade I, Bellefontaine R, Guinko S, Lykke AM, Diallo A, Boussim JI. 2008. Identification des arbres hors forêt préférés des populations du Sanmatenga (Burkina Faso). Bois et Forêts des Tropiques, 298 (4) : 53-64. DOI :

https://doi.org/10.19182/bft2008.298.a20 366

Belem B. 2009. Ethnobotanique et conservation de Bombax costatum
Pellegr. Et Vuillet (kapokier à fleurs rouges) dans les systèmes de production agricoles du plateau central, Burkina Faso. Thèse unique de Doctorat, Univ. de Ouagadougou, 143 p+ annexes.

Boffa JM, Taonda SJB, Dicket JB, Knudson DM. 2000. Field-scale influence of karité (Vitellaria paradoxa) on sorghum production in the Sudan zone of Burkina Faso. Agrof. Syst., 49: 153-175. DOI: http://dx.doi.org/10.1023/A:1006389828 259.

Coulibaly-Lingani P, Savadogo P, MulualemTigabu M, Oden PC. 2011. Factors influencing people's participation in the forest management program in Burkina Faso, West Africa. Forest Policy and Economics, Elsevier, vol. 13(4): 292302. DOI: 10.1016/j.forpol.2011.02.005.

Elias M, Carney J. 2007. African shea butter: A feminized subsidy from nature. Africa, 77: 37-62. DOI: 10.1353 / afr.2007.0018

Fontès J, Guinko S. 1995. Carte de la végétation et de l'occupation du sol du Burkina Faso : Notice explicative. Toulouse : Ministère de la coopération Française 66p.

Gnanglè PC, Egah J, Baco MN, Gbemavo CDSJ, Kakaï RG, Sokpon N. 2012. Perceptions locales du changement climatique et mesures d'adaptation dans la gestion des parcs à karité au NordBénin. Int. J. Biol. Chem. Sci., 6(1): 136149, 2012.2 DOI: http://dx.doi.org/10.4314/ijbcs.v7i3.16.

Kabore SA, Bastide B, Traore S, Boussim JI. 2012. Dynamique du karité, Vitellaria paradoxa, dans les systèmes agraires du Burkina Faso. Bois et Forêts des Tropiques, 313(3): 47-59. DOI : https://doi.org/10.19182/bft2012.313.a20 496

Kristensen M, Balslev H. 2003. Perceptions, use and availability of woody plants 
among the Gourounsi in Burkina Faso. Biodiversity and conservation, 12: 17151739.

DOI: https://doi.org/10.1023/A:102361481687 8

Kristensen M, Lykke AM. 2003. InformantBased Valuation of Use and Conservation Preferences of Savanna Trees in Burkina Faso. Economic Botany, 57(2):203-217. 2003. DOI: https://doi.org/10.1663/00130001(2003)057

[0203: IVOUAC]2.0.CO;2

Lamy Lamy GM, Ibrahima A, Ndjonka D, Mapongmetsem P. 2018. Etude ethnobotanique des sous-variétés de Syzygium guineense (Will.) DC. var. macrocarpum (Engl.) F. White dans les Hautes Savanes Guinéennes (Adamaoua, Cameroun). Int. J. Biol. Chem. Sci., 12(4): 1636-1649. DOI: https://dx.doi.org/10.4314/ijbcs.v12i4.11

Lovett PN, Haq N. 2000. Evidence for anthropic selection of the Shea nut tree (Vitellaria paradoxa). Agroforestry Systems, 48: 273-288. DOI: 10.1023 / A: 1006379217851

Lykke AM, Kristensen MK, Ganaba S. 2004. Valuation of local use and dynamics of 56 woody species in the Sahel. Biodiversity and Conservation, 13: 1961-1990. DOI: https://doi.org/10.1023/B:BIOC.0000035 876.39587.1a

Mapongmetsem PM, Nkongmeneck BA, Rongoumi G, Dongock DN, Dongmo EB. 2011. Impact des systèmes d'utilisation des terres sur la conservation de Vitellaria paradoxa Gaerten. F. (Sapotaceae) dans la région des savanes soudano guinéennes. International Journal of Environmental Studies, $\quad \mathbf{6 8}(6)$ : $\quad 851-872 . \quad$ DOI: 10.1080/00207233.2011.587259
Mertz O, Lykke AM, Reenberg A. 2001. Importance and seasonality of vegetable consumption and marketing in Burkina Faso. Economic Botany, 55(2): 276-289. DOI :

https://doi.org/10.1007/BF02864565

Orwa C, Mutua A, Kindt R, Jamnadass R, Anthony S. 2009. Agroforestree Database : a tree reference and selection guide version 4.0. World Agroforestry Center.

Kenya.

http://www.worldagroforestry.org/resour ces/databases/agroforestree

PRPW/ECOPAS. 2002. Mission d'étude de la vegetation (aspects ethnobotaniques) du complexe écologique du Parc W. Rapport définitif. ECOPAS, Ouagadougou $44 \mathrm{p}$.

Smektala G, Peltier R, Sibelet N, Leroy M, Manlay R, Njiti CF, Ntoupka M, Njiemoun A, Palou, Tapsou O. 2005. Parcs agroforestiers sahéliens : de la conservation à l'aménagement. Vertig $O$ la revue électronique en sciences de l'environnement [En ligne], 6(2): mis en ligne le 01 septembre 2005, consulté le 17 février 2019. DOI: https://dx.doi.org/10.4000/vertigo.4410.

Thiombiano A, Bonkoungou EG, Sawadogo L, Ouadba JM, Boussim JI, Ganaba S, Diallo OB, Sina S, Ouédraogo K, Kaboré C, Coulibaly/Lingani P, Zida D, Soulama S. 2016. Etat des peuplements et du développement des technologies pour une meilleure productivité du karité au Burkina Faso. CORAF/INERA, Ouagadougou, 76p.

Thiombiano A, Schmidt M, Dressler S, Ouédraogo A, Hahn K, Zizka G. 2012. Catalogue des Plantes Vasculaire du Burkina Faso. Boissiera / Conservatoire et Jardin Botaniques de la ville de Genève. Volume 65; 391 p. 
Tiétiambou FRS, Lykke AM, Korbéogo G, Thiombiano A, Ouédraogo A. 2016. Perceptions et savoirs locaux sur les espèces oléagineuses locales dans le Kénédougou, Burkina Faso. Bois et Forêts des Tropiques, 327 (1) : 39-50. DOI :

https://doi.org/10.19182/bft2016.327.a31 295

Traoré L, Ouédraogo I, Ouédraogo A, Thiombiano A. 2011. Perceptions, usages et vulnérabilité des ressources végétales ligneuses dans le Sud-Ouest du Burkina Faso. Int. J. Biol. Chem. Sci., 5(1): 258-278. DOI : http://dx.doi.org/10.4314/ijbcs.v5i1.6810 3

War H. 2007. La gestion participative et le développement des PFNL comme moyen de réduction de la pauvreté féminine en zone rurale : cas du Maghreb et du Sahel. Mémoire de DESS en Gestion de la Politique Economique (GPE), Université de Cocody, Abidjan, République de la Côte d'Ivoire. 60p.
Yaméogo G, Yélémou B, Boussim IJ, Traoré D. 2013. Gestion du parc agroforestier du terroir de Vipalogo (Burkina Faso) : contribution des ligneux à la satisfaction des besoins des populations. Int. J. Biol. Chem. Sci., 7(3) : 1087-1105. DOI : http://dx.doi.org/10.4314/ijbcs.v7i3.16.

Yaméogo G, Yélémou B, Traoré D. 2005. Pratique et perceptions paysannes dans la création de parc agroforestier dans le terroir de Vipalogo (Burkina Faso). Biotechnol. Agron. Soc. Environ, 9 (4) : 241-248.

Yaméogo JT, Hien M, Lykke AM, Somé AN, Thiombiano A. 2011. Effet des techniques de conservation des eaux et des sols, zaï forestier et cordons pierreux, sur la réhabilitation de la végétation herbacée à l'Ouest du Burkina Faso. Int. J. Biol. Chem. Sci., 5(1): 5671.

DOI : http://dx.doi.org/10.4314/ijbcs.v5i1.6808 5 